\title{
Use of check-point inhibitors in the treatment of progressive multifocal leukoencephalopathy
}

\author{
Luis Miguel Juárez-Salcedo ${ }^{1}$, Samir Dalia ${ }^{2}$ \\ ${ }^{1}$ Hematology Department, Gregorio Marañón University Hospital, Madrid, Spain; ${ }^{2}$ Hematology/Oncology, Mercy Clinic Oncology and Hematology \\ - Joplin, Missouri, USA \\ Correspondence to: Samir Dalia, MD. Hematology/Oncology, Mercy Clinic Oncology and Hematology - Joplin, Missouri, USA. \\ Email: sdalia@gmail.com; Luis Miguel Juárez-Salcedo, MD. Hematology Department, Gregorio Marañón University Hospital, Madrid, Spain. \\ Email: Dr.luisjuarez@gmail.com. \\ Provenance: This is an invited article commissioned by the Academic Editor Dr. Zhenxiang Zhao (Department of Neurology, Henan Provincial \\ People's Hospital, People's Hospital of Zhengzhou University, People's Hospital of Henan University, Zhengzhou, China). \\ Comment on: Cortese I, Muranski P, Enose-Akahata Y, et al. Pembrolizumab Treatment for Progressive Multifocal Leukoencephalopathy. N Engl J \\ Med 2019;380:1597-605.
}

Submitted Oct 22, 2019. Accepted for publication Oct 31, 2019.

doi: $10.21037 /$ atm.2019.11.11

View this article at: http://dx.doi.org/10.21037/atm.2019.11.11

Progressive multifocal leukoencephalopathy (PML) is an opportunist viral infection predominantly seen in immunosuppressed patients (most commonly in HIV cases, lymphoproliferative disorders and primary immunodeficiency) $(1,2)$. This aggressive brain infection is caused by the JC virus (JCV), a DNV virus of the human polyomavirus family (3). Primary viremia is usually asymptomatic and occurs in childhood. After the primary infection, the virus remains in the body in a latent stage $(4,5)$. The reactivation can be frequently following immunosuppression or after elimination of the B cells with drugs targeting $\mathrm{B}$ cells such a as monoclonal antibodies $(\mathrm{mAb})(6)$.

The conventional treatment of PML include intense antiretroviral therapy like Cidofovir, with little response rates. Mofloquine, an anti-malaria treatment (7), and Mirtazapine, an inhibitor of 5-HT2a receptor (8), have demonstrated efficacy when human glial cell line is infected.

The significant role of cytotoxic $\mathrm{T}$ lymphocytes directed against JCV in controlling the disease has been established and is indicated by the correlation between an impaired Th-1 type T helper function and clinical manifestation (9). In the era of targeted therapies, the search for drugs that act on signaling pathways are considered as viable options.

Programmed cell death protein $1(\mathrm{PD}-1)$ is an inhibitory T-cell surface receptor that keeps the immune system from attacking the body's own tissues and is a marker of T-cell exhaustion. PD-1 expression is elevated on the CD4+ and CD8+ T lymphocytes of patients with PML and is particularly elevated on JC virus-specific CD8+ T cells (10). The blocking of the PD-1 receptor increases the cellular immune response to JC virus in healthy volunteers but only if detectable JC virus-specific CD8+ T cells are present at baseline.

Pembrolizumab, an immune checkpoint inhibitor, is a humanized monoclonal antibody that blocks the interaction between PD-1 and its ligands, PD-L1 and PD-L2. Use of this drug to reinvigorate anti-JC virus immune activity in patients with PML was unknown.

Cortese et al., have published the results of a study of 8 patients diagnosed with PML and who have been treated with Pembrolizumab. These patients received this drug at dose of $2 \mathrm{mg}$ per kilogram of body weight, administered intravenously, every 4 to 6 weeks. Seven of the patients presented with neurological symptoms. All patients had JC viral load in the cerebral spinal fluid (CSF). Patients' immune conditions included immunodeficiency associated to HIV infection, distinct hematological diseases and idiopathic lymphopenia. All of them presented with abnormal CD4+, CD8+ and CD19+ cell counts. Patients all received a minimum of two doses of pembrolizumab. Serial neurological examination, MRI and CSF sampling 
were used for the follow-up. After treatment with Pembrolizumab, five of the eight patients had clinical improvement or stabilization, and no patient had complete disappearance of PML lesions. The five patients that presented any grade of response had a reduction in JC viral load in the CSF. Four out of the five patients showed a persistent reduction in JC viral load and clinical stabilization 16 to 26 months after receiving treatment (11).

The results of this study show a possibility that pembrolizumab may help treat refractory JC virus infections. There are a couple of points to note about this case series prior to drawing any generalized conclusions. First, the number of injections received varied among the patients, making it difficult to relate the improvement of symptoms with a greater number of injections received. It is still unclear how many doses of this medication should be given to try to treat JC virus. Two HIV-patients reported better outcomes and this may be due to their antiretroviral medications which leads to immune recovery and may not be due to the pembrolizumab. Further, two patients with CLL and Hodgkin's lymphoma had stabilization of previous neurological symptoms associated with PML 1 month after Pembrolizumab treatment but did not have a clear initial benefit to the medication.

This case series shows that pembrolizumab may be a potential treatment for patients with PML. Larger systematically conducted studies are need to confirm if pembrolizumab may be a potential future treatment for JCV reactivation in certain populations. We are hopeful that future clinical trials will be performed in the different populations effected by JCV reactivation.

\section{Acknowledgments}

None.

\section{Footnote}

Conflicts of Interest: The authors have no conflicts of interest to declare.

Ethical Statement: The authors are accountable for all aspects of the work in ensuring that questions related to the accuracy or integrity of any part of the work are appropriately investigated and resolved.

\section{References}

1. Brooks BR, Walker DL. Progressive multifocal leukoencephalopathy. Neurol Clin 1984;2:299-313.

2. Bernick C, Gregorios JB. Progressive multifocal leukoencephalopathy in a patient with acquired immune deficiency syndrome. Arch Neurol 1984;41:780-2.

3. ZuRhein G, Chou SM. Particles resembling papova viruses in human cerebral demyelinating disease. Science 1965;148:1477-9.

4. Calderelli-Stefano R, Vago L, Omodeo-Zorini E, et al. Detection and typing of JC virus in autopsy brains and extraneural organs of AIDS patients and non-immunocompromised individuals. J Neurovirol 1999;5:125-33.

5. Jensen PN, Major EO. Viral variant nucleotide sequences help expose leukocytic positioning in the JC virus pathway to the CNS. J Leukoc Biol 1999;65:428-38.

6. Bohra C, Sokol L, Dalia S. Progressive Multifocal Leukoencephalopathy and Monoclonal Antibodies: A Review. Cancer Control 2017;24:1073274817729901.

7. Brickelmaier M, Allaire NE, Gorelik L. Identification and characterization of Mefloquine efficacy against JC Virus in vitro. Mult Scler 2008;14:S44.

8. Verma S, Cikurel K, Koralnik IJ, et al. Mirtazapine in progressive multifocal leukoencephalopathy associated with polycythemia vera. J Infect Dis 2007;196:709-11.

9. Weber F, Goldmann C, Krämer M, et al. Cellular and humoral immune response in progressive multifocal leukoencephalopathy. Ann Neurol 2001;49:636-42.

10. Tan CS, Bord E, Broge TA Jr, et al. Increased program cell death-1 expression on $\mathrm{T}$ lymphocytes of patients with progressive multifocal leukoencephalopathy. J Acquir Immune Defic Syndr 2012;60:244-8.

11. Cortese I, Muranski P, Enose-Akahata Y, et al. Pembrolizumab Treatment for Progressive Multifocal Leukoencephalopathy. N Engl J Med 2019;380:1597-605.
Cite this article as: Juárez-Salcedo LM, Dalia S. Use of checkpoint inhibitors in the treatment of progressive multifocal leukoencephalopathy. Ann Transl Med 2019;7(Suppl 8):S297. doi: 10.21037/atm.2019.11.11 